\title{
Pulmonary hamartoma resembling multiple metastases: A case report
}

\author{
ZHENYA LU $^{1}$, FANGFANG QIAN ${ }^{2}$, SHANWEN CHEN $^{3}$ and GUOWEI YU ${ }^{4}$ \\ ${ }^{1}$ Department of Internal Medicine, The First Affiliated Hospital, School of Medicine, Zhejiang University, \\ Hangzhou, Zhejiang 310003; ${ }^{2}$ The First Clinical Medicine, Wenzhou Medical University, Wenzhou, \\ Zhejiang 325000; Departments of ${ }^{3}$ Urology and ${ }^{4}$ Thoracic Surgery, The First Affiliated Hospital, \\ School of Medicine, Zhejiang University, Hangzhou, Zhejiang 310003, P.R. China
}

Received August 21, 2013; Accepted February 26, 2014

DOI: $10.3892 / 01.2014 .2043$

\begin{abstract}
The current study presents the case of a patient with multiple pulmonary nodules as observed by computed tomography. Furthermore, a marginal increase in fluorodeoxyglucose uptake was identified by positron emission tomography. Due to the appearance of multiple small nodules and a history of radical nephrectomy, a hypothetical diagnosis of pulmonary metastasis of a previously excised renal carcinoma was determined, which was confirmed by biopsy. Video-assisted thoracoscopic surgical resection of the nodules was proposed and pathological examination exhibited an unforeseen and rare observation.
\end{abstract}

\section{Introduction}

Pulmonary metastasis is a common occurrence in patients with renal cancer and is usually treated with immunotherapy and novel agents that target angiogenesis (1). Certain clinical studies have indicated that the resection of pulmonary metastases (metastasectomy) may be a treatment option (1-2). However, the role of surgery for metastases originating from renal cancer has yet to be fully determined. Pulmonary nodules that appear in patients who have undergone nephrectomy for renal cancer are usually pulmonary metastases. However, the occurrence of metachronous lung tumors and certain benign diseases, particularly pulmonary hamartoma, are uncommon (3). The patient provided written informed consent.

Correspondence to: Dr Shanwen Chen, Department of Urology, The First Affiliated Hospital, School of Medicine, Zhejiang University, 79 Qingchun Road, Hangzhou, Zhejiang 310003, P.R. China

E-mail: chensw123@126.com

Key words: pulmonary hamartoma, resemble, metastasis

\section{Case report}

A 55-year-old female was referred to the First Affiliated Hospital, School of Medicine of Zhejiang University (Hangzhou, China) due to the presence of multiple round pulmonary nodules on a chest computed tomography (CT) scan, which was performed during a postoperative follow-up evaluation for renal cancer. The patient reported no history of cough, fever, chest pain, dyspnea, hemoptysis, weight loss or tuberculosis. In addition, no peripheral lymphadenopathy was detected and the routine blood test results, including a hemogram and renal and liver function tests, were within the normal ranges.

The patient underwent a contrast-enhanced $\mathrm{CT}$ of the chest, which revealed multiple round pulmonary nodules measuring $\sim 10 \times 12 \mathrm{~mm}$ with clear boundaries in the lungs (Fig. 1). In addition, an ${ }^{18} \mathrm{~F}$-fluoro-2-deoxy-D-glucose (FDG)-positron emission tomography (PET)/CT scan was performed to characterize the nodules, which exhibited a mild uptake of FDG that is indicative of malignancy (Fig. 2). In addition, bronchoscopy showed normal bronchi. Due to the presence of multiple small nodules with clear boundaries in the lungs and the history of a radical nephrectomy, a hypothetical diagnosis of pulmonary metastasis of a previously removed renal carcinoma was determined and subsequently confirmed by biopsy.

A video-assisted thoracoscopic nodulectomy was performed on the patient and frozen-section analysis revealed that the tumor was benign (possibly a pulmonary hamartoma) and the procedure was terminated.

The anatomopathological examination revealed that the mass was a non-capsulated, regular lesion measuring $10 \times 11 \times 12 \mathrm{~mm}$, with a firm and fibroelastic consistency. In addition, microscopic analysis revealed blood vessels, well-differentiated adipose tissue and polygonal cells (Fig. 3). Immunohistochemistry revealed pan-cytokeratin (-), melan-A (+), HMB45 (-), HHF35 (-), p53 (-), S-100 (+), desmin (-), cluster of differentiation $68(-)$ and smooth muscle actin (+) expression, which is consistent with pulmonary hamartoma.

The patient recovered well and was discharged on the third postoperative day. After six months of postoperative follow-up, 


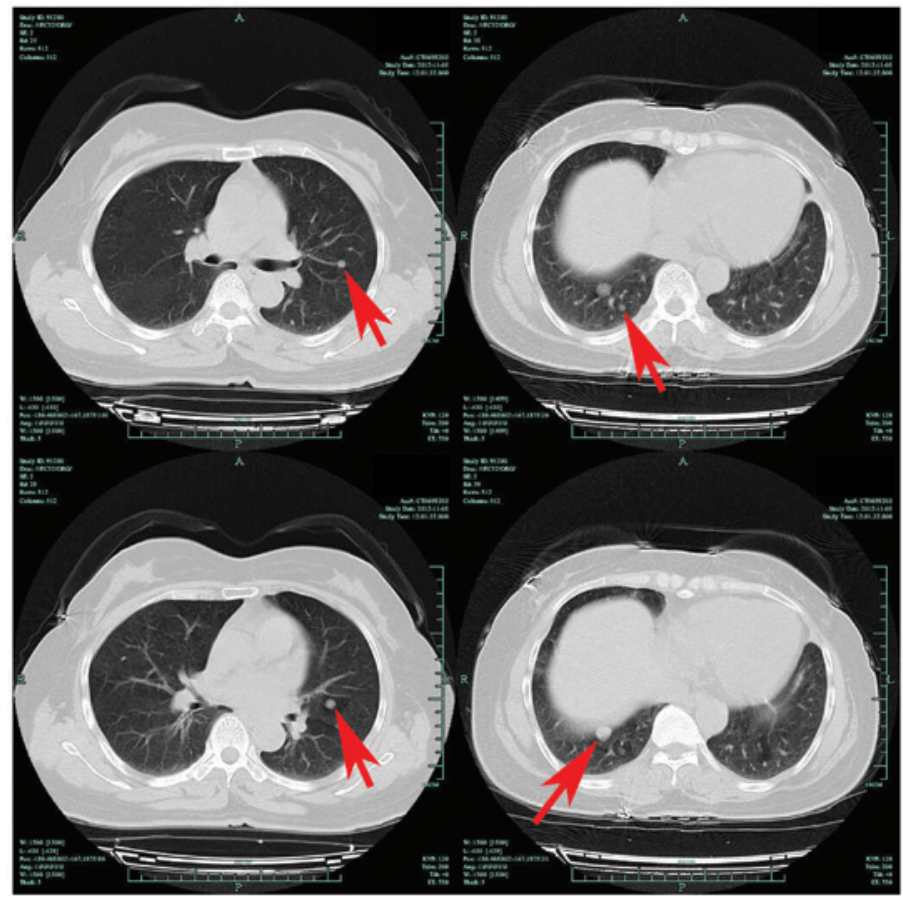

Figure 1. Contrast-enhanced computed tomography of the chest revealed multiple round pulmonary nodules, measuring $10 x 12 \mathrm{~mm}$ with clear boundaries, in the lungs (indicated by the arrows).
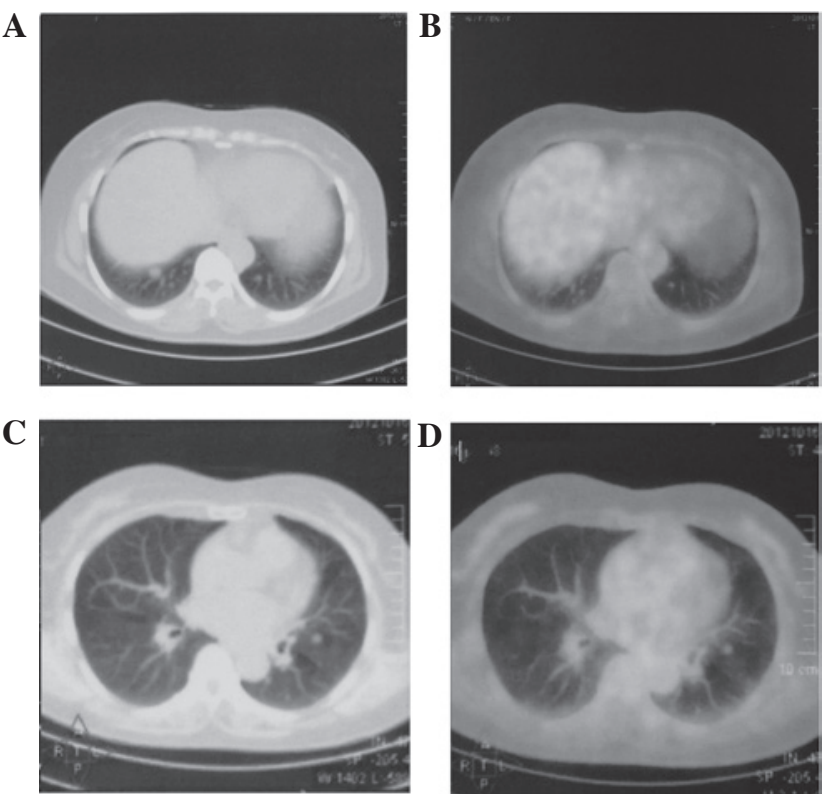

Figure 2. (A and C) Computed tomography (CT) and (B and D) ${ }^{18} \mathrm{~F}$-fluoro-2-deoxy-D-glucose (FDG)-positron emission tomography/CT scan images exhibit a mild uptake of ${ }^{18} \mathrm{~F}-\mathrm{FDG}$ in the pulmonary nodule indicative of malignancy.

the patient has presented no signs of increasing multiple pulmonary nodules as assessed by computed tomography.

\section{Discussion}

Pulmonary hamartoma account for $77 \%$ of all benign lung tumors and $4 \%$ of all solitary lung nodules (4-5). The lesion has been described as a benign neoplasm of the fibrous connective tissue of the bronchi surrounded by respiratory epithelium that

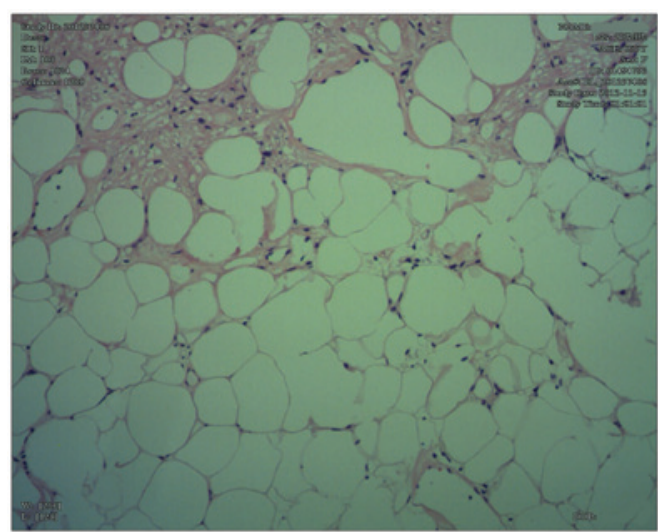

Figure 3. A section obtained from the pulmonary mass showed blood vessels, well-differentiated adipose tissue and polygonal cells. (stain, hematoxylin and eosin; magnification, x100).

commonly contains cartilage and adipose tissue, which does not comply with the usual histological distribution of the lung (6). In total, $90 \%$ of the hamartomas manifest as a solitary peripheral mass (5) and rarely occur in the form of multiple lesions (7). In addition, hartoma is more common in adults and the incidence rate is twice as high in males compared with females. The mean growth rate of hamartoma is $3.2 \pm 2.6 \mathrm{~mm} /$ year (8) and the occurrence of malignancy in hamartoma patients is possible. Certain studies have found that the incidence of bronchial carcinoma is 6.3-fold higher in patients with hamartoma than in a normal population, indicating the presence of an etiologic association (9). The appearance of pulmonary nodules during the follow-up evaluation of patients who have undergone nephrectomy is often confusing. Nine patients with a history of radical nephrectomy for renal cell carcinoma underwent the surgical removal of newly detected pulmonary nodules at the Hiroshima 
University Hospital (Hiroshima, Japan). Of these nine patients, six had metastatic lung tumors, two had bronchogenic primary carcinomas and one had a granulomatous infection (10).

The diagnostic algorithm in pulmonary hamartomas usually begins with structural imaging studies. Chest X-ray and CT are useful, however, magnetic resonance imaging has a limited role. Hamartomas are benign lesions containing normal pulmonary tissue and $\mathrm{CT}$ observations, such as internal fat or popcorn-like calcifications, are useful for distinguishing hamartomas from other malignancies (11-12). Certain studies have also demonstrated the presence of adipose tissue in $50 \%$ of the hamartomas that were evaluated by computed tomography (10). Radiological differentiation between benign and malignant nodules is determined according to size, margins, contour and internal characteristics, however, the interpretation may be fallacious (11-13). For example, in the present case, the lesion did not exhibit any such features on the CT scan. The CT also failed to reveal any signs of associated pulmonary tuberculosis.

FDG-PET scan is a useful non-invasive assessment in the differential diagnosis of indeterminate lung lesions, particularly in cases with an intermediate risk of malignancy (14). False-positive results from FDG-PET have been associated with focal infections or inflammatory conditions. In a previous study, six patients with pulmonary hamartoma underwent FDG-PET and only one demonstrated an accumulation of FDG (15-16). Tumors with a low metabolic rate, such as bronchioloalveolar carcinomas or carcinoids, may result in false-negative results, although, more recent results often describe mild FDG uptake in carcinoid lesions (17-18). Scott et al (19) also reported false-negative results in two patients with very small tumors. The rate of glycolysis in the tumor may have resulted in the low rate of FDG uptake and the actual amount of FDG uptake by the malignant tissue may have been relatively small, which resulted in a low overall FDG uptake. Furthermore, false-negative results are also possible in small tumors due to partial volume effects.

As the preoperative diagnosis of pulmonary hamartoma is often difficult, surgical resection is required for the differential diagnosis of lung cancer or metastatic lung tumors, unless clinical imaging reveals typical observations of pulmonary hamartoma. In the present study, considering the presence of multiple pulmonary nodules and the patient's history of radical nephrectomy, metastasis was suggested as the initial diagnosis. Therefore, the histopathological diagnosis of hamartoma was unpredicted. The pulmonary nodules presented in patients who have undergone nephrectomy for renal cancer are not always pulmonary metastases and the confirmation of the histopathological diagnosis is fundamentally important to determine the optimal treatment method.

In conclusion. bronchoscopy with a biopsy is recommended for endobronchial lesions, as well as for patients with pulmonary symptoms, such as a cough, hemoptysis, recurrent pulmonary infections or atelectasis (20). In addition, percutaneous transthoracic aspiration biopsy diagnoses $85 \%$ of hamartomas, that present close to the thoracic wall, by differentiating them from nodules of other etiologies, such as renal cancer lung metastasis. Despite thorough clinical assessment with advanced imaging technology and needle biopsy, a number of patients continue to undergo surgery for benign disease. Therefore, future studies are required to identify novel strategies for the diagnosis and treatment of early-stage lung cancer (21). In cases where a diagnosis has not been determined due to the stiffness of the tumor, rendering a percutaneous biopsy useless, enucleation or resection via open thoracotomy or video-assisted resection is recommended (22).

\section{Acknowledgements}

The present study was supported by a grant from the National Key Clinical Specialty Construction Project of China.

\section{References}

1. Tsakiridis K, Visouli AN,Zarogoulidis P,Mpakas A, Machairiotis N, Stylianaki A, Katsikogiannis N, Courcoutsakis N and Zarogoulidis K: Lost in time pulmonary metastases of renal cell carcinoma: complete surgical resection of metachronous metastases, 18 and 15 years after nephrectomy. J Thorac Dis 4 (Suppl 1): S69-S73, 2012.

2. Oddsson SJ, Hardarson S, Petursdottir V, Jonsson E, Sigurdsson MI, Einarsson GV, Pfannschmidt J and Gudbjartsson T: Synchronous pulmonary metastases from renal cell carcinoma - a whole nation study on prevalence and potential resectability. Scand J Surg 101: 160-165, 2012.

3. Hamano A, Yamashita Y, Yumura Y, Takase K, Ogo Y, Noguchi S, Morohoshi T, Satomi Y and Fukuda M: Three cases of pulmonary hamartoma appearing after radical nephrectomy for renal cell carcinoma. Hinyokika Kiyo 51: 805-807, 2005 (In Japanese).

4. Arrigoni MG, Woolner LB, Bernatz PE, Miller WE and Fontana RS: Benign tumors of the lung. A ten-year surgical experience. J Thorac Cardiovasc Surg 60: 589-599, 1970.

5. Khouri NF, Meziane MA, Zerhouni EA, Fishman EK and Siegelman SS: The solitary pulmonary nodule. Assessment, diagnosis, and management. Chest 91: 128-133, 1987.

6. Bateson EM: So-called hamartoma of the lung - a true neoplasm of fibrous connective tissue of the bronchi. Cancer 31: 1458-1467, 1973.

7. Bennett LL, Lesar MS and Tellis CJ: Multiple calcified chondrohamartomas of the lung: CT appearance. J Comput Assist Tomogr 9: 180-182, 1985.

8. Hansen CP, Holtveg H, Francis D, Rasch L and Bertelsen S: Pulmonary hamartoma. J Thorac Cardiovasc Surg 104: 674-678, 1992.

9. Karasik A, Modan M, Jacob CO and Lieberman Y: Increased risk of lung cancer in patients with chondromatous hamartoma. J Thorac Cardiovasc Surg 80: 217-220, 1980.

10. Nakamoto T, Igawa M, Mitani S, Usui A, Yoshioka S, Nishiki M and Usui T: Pulmonary nodules in patients with a history of radical nephrectomy for renal cell carcinoma. Int J Urol 2: 229-231, 1995.

11. Diederich S and Das M: Solitary pulmonary nodule: detection and management. Cancer Imaging 6: S42-S46, 2006.

12. Wahidi MM, Govert JA, Goudar RK, Gould MK, McCrory DC; American College of Chest Physicians: Evidence for the treatment of patients with pulmonary nodules: when is it lung cancer? ACCP evidence-based clinical practice guidelines (2nd edition). Chest 132 (3 Suppl): S94-S107, 2007.

13. Jeong YJ, Yi CA and Lee KS: Solitary pulmonary nodules: Detection, characterization, and guidance for further diagnostic workup and treatment. AJR Am J Roentgenol 188: 57-68, 2007.

14. Gould MK, Fletcher J, Iannettoni MD, Lynch WR, Midthun DE, Naidich DP, Ost DE; American College of Chest Physicians: Evaluation of patients with pulmonary nodules: when is it lung cancer? ACCP evidence-based clinical practice guidelines (2nd edition). Chest 132 (3 Suppl): S108-S130, 2007.

15. Sminohara S, Hanagiri T, Kuwata T, Takcenaka M, Oka S, Chikainsi Y, Nagata Y,Shimokawa H, Shigematsu Y, Nakagawa M, et al: Clinical characteristics of pulmonary hamartoma resected surgically as undiagnosed pulmonary nodule. J UOEH 34: 41-46, 2012 (In Japanese). 
16. Okagawa T, Uchida T and Suyama M: Endobronchial hamartoma suspected lung cancer due to false positive of fluorodeoxyglucose-positron emission tomography; report of a case. Kyobu Geka 62: 833-835, 2009 (In Japanese).

17. Zhang LN, Xue XY, Wang $N$ and Wang JX: Mimicking pulmonary multiple metastatic tumors: A case of primary nodular parenchymal pulmonary amyloidosis with review of the literature. Oncol Lett 4: 1366-1370, 2012.

18. Daniels CE, Lowe VJ, Aubry MC, Allen MS and Jett JR: The utility of fluorodeoxyglucose positron emission tomography in the evaluation of carcinoid tumors presenting as pulmonary nodules. Chest 131: 255-260, 2007.

19. Scott WJ, Schwabe JL, Gupta NC, et al: Positron emission tomography of lung tumors and mediastinal lymph nodes using [18F]fluorodeoxyglucose. The Members of the PET-Lung Tumor Study Group. Ann Thorac Surg 58: 698-703, 1994.
20. Cosío BG, Villena V, Echave-Sustaeta J, de Miguel E, Alfaro J, Hernandez L and Sotelo T: Endobronchial hamartoma. Chest 122: 202-205, 2002.

21. Smith MA, Battafarano RJ, Meyers BF, Zoole JB, Cooper JD and Patterson GA: Prevalence of benign disease in patients undergoing resection for suspected lung cancer. Ann Thorac Surg 81: 1824-1828, 2006.

22. Ramming KP: Surgery for pulmonary metastases. Surg Clin North Am 60: 815-824, 1980. 